\title{
An Investigation of the Morphology of the Petrotympanic Fissure Using Cone-Beam Computed Tomography
}

\author{
Spyros Damaskos ${ }^{1,2}$, Konstantinos Syriopoulos ${ }^{3,4}$, Rogier L. Sens ${ }^{3,4}$, Constantinus Politis ${ }^{3,4}$ \\ ${ }^{1}$ Department of Oral Radiology, Academic Centre for Dentistry Amsterdam, Amsterdam, The Netherlands. \\ ${ }^{2}$ Department of Oral Diagnosis and Radiology, School of Dentistry, NKUA, Athens, Greece. \\ ${ }^{3}$ Department of Imaging and Pathology, OMFS IMPATH Research Group, Faculty of Medicine, Katholieke Universiteit \\ Leuven, Leuven, Belgium. \\ ${ }^{4}$ Department of Oral and Maxillofacial Surgery, University Hospitals Leuven, Leuven, Belgium.
}

\author{
Corresponding Author: \\ Spyros Damaskos \\ Department of Oral Radiology \\ Academic Centre for Dentistry Amsterdam (ACTA) \\ Gustav Mahler Laan 3004, 1081 LA, Amsterdam \\ The Netherlands \\ Phone: +30 6944541529 \\ E-mail: s.damaskos@acta.nl;sdamask@dent.uoa.gr
}

\begin{abstract}
Objectives: The purpose of the present study was: a) to examine the visibility and morphology of the petrotympanic fissure on cone-beam computed tomography images, and b) to investigate whether the petrotympanic fissure morphology is significantly affected by gender and age, or not.

Material and Methods: Using Newtom VGi (QR Verona, Italy), 106 cone-beam computed tomography examinations (212 temporomandibular joint areas) of both genders were retrospectively and randomly selected. Two observers examined the images and subsequently classified by consensus the petrotympanic fissure morphology into the following three types: type 1 - widely open; type 2 - narrow middle; type 3 - very narrow/closed.

Results: The petrotympanic fissure morphology was assessed as type 1, type 2, and type 3 in 85 (40.1\%), 72 (34.0\%), and $55(25.9 \%)$ cases, respectively. No significant difference was found between left and right petrotympanic fissure morphology $($ Kappa $=0.37 ; \mathrm{P}<0.001)$. Furthermore, no significant difference was found between genders, specifically $\mathrm{P}=0.264$ and $\mathrm{P}=$ 0.211 for the right and left petrotympanic fissure morphology, respectively. However, the ordinal logistic regression analysis showed that males tend to have narrower petrotympanic fissures, in particular $\mathrm{OR}=1.58$ for right and $\mathrm{OR}=1.5$ for left petrotympanic fissure.

Conclusions: The current study lends support to the conclusion that an enhanced multi-planar cone-beam computed tomography yields a clear depiction of the petrotympanic fissure's morphological characteristics. We have found that the morphology is neither gender nor age-related.
\end{abstract}

Keywords: arthroscopy; cone-beam computed tomography; temporal bone; temporomandibular joint.

Accepted for publication: 27 March 2018

To cite this article:

Damaskos S, Syriopoulos K, Sens RL, Politis C.

An Investigation of the Morphology of the Petrotympanic Fissure Using Cone-Beam Computed Tomography

J Oral Maxillofac Res 2018;9(1):e4

URL: http://www.ejomr.org/JOMR/archives/2018/1/e4/v9n1e4.pdf

doi: $10.5037 /$ jomr.2018.9104 


\section{INTRODUCTION}

Arthroscopic surgery of the temporomandibular joint (TMJ) involves puncturing the superior joint compartment with a trocar and subsequently the examination with an arthroscope. Complications arising from an inadvertent trauma to the surrounding anatomical structures may include injury to the external ear, the middle ear $[1,2]$, and even the brain $[\underline{3}, \underline{4}]$ because the roof of the glenoid fossa is very thin and translucent [ $\underline{5}$. For 301 cases of arthroscopic lysis and lavage, Tsuyama et al. [] have reported an incidence of $8.6 \%$ of otologic complications.

During the surgery, arthroscopic anatomy does not allow a surgical view of the fissures underlying the fibrocartilage [7], or ruptures in the bone of the fossa. This applies particularly when prior to the scope's entry the first puncture is placed at the maximum concavity of the glenoid fossa. During the TMJ arthroscopy, Sugisaki et al. [] $]$ indicated the potential risk of the middle ear injury, through the foramen of Huschke and/or Hugier's canal, of the tympanic plate. Hugier's canal is at the medial end of the petrotympanic fissure (PTF), through which the chorda tympani nerve exits the tympanic cavity (alsocalled "canal of Huguier", or "iter chordae anterius"). Furthermore, in 1962 Pinto [9] showed that there is a close relationship between the structures of TMJ and the middle ear. This was mainly attributed to the presence of the anterior malleolar ligament (AML), and the disco malleolar ligament (DML) [10-12]. Both ligaments exit the tympanic wall anteriorly from the malleus' anterior process directed towards the PTF [13-16]. DML and AML are separated in the PTF by an osseous triangular crest. He also suggested that the movements of TMJ transferred via DML could cause the movement of the ear ossicles and the tympanic membrane [9]. A recent histological study supports this assumption [14]. These movements could cause subjective hearing loss, tinnitus and TMJ pain [17]. Most recently, Buergers et al. [17] showed that the prevalence of tinnitus was found to be 8 times higher in participants with temporomandibular disorders (TMD) than in participants without TMD. Moreover, some studies suggest that the degree of closure of PTF could explain the presence of hearing symptoms in patients with TMD $[\underline{11}, \underline{16}]$. Çakur et al. [18] found that a statistically important negative association exists between PTF type and tinnitus. They also state that a short, wide PTF (type 1) might be associated with an increased risk of tinnitus in patients with TMD [18]. Additionally, Schickinger et al. [19] reported that a variation of the PTF morphology could be a possible cause of an otologic complication during TMJ arthroscopy.

A comprehensive anatomic review of the fissures related to TMJ structure was published by Anagnostopoulou et al. [20]. However, that study was based on 40 dry skulls and no cone-beam computed tomography (CBCT) evaluation was performed.

This delicate bony anatomical structure of PTF can neither be viewed directly during surgery, nor can that structure be properly assessed by means of panoramic radiographs, due to their inherent limitations. Thus, the preoperative CBCT or computed tomography (CT) remain the only means of assessing continuity of the bony lining of the fossa, and the abnormal presence of ruptures and fissures in the bony cavity of the joint. By using short scanning times and reduced patient dose, the CBCT systems' advantage is that they produce highquality images with submillimetre resolution. CBCT may also provide an accurate depiction of subtle structures, such as: a) the surgically relevant middle ear structures, b) the anatomy of the temporal bone, and c) the lateral skull base. That accurate depiction is comparable to those of multi-detector computed tomography (MDCT) [21,22]. Consequently, a preoperative CBCT examination could reveal an open PTF. This finding could be considered as a "locus minoris resistentiae" and thus constituting a possible contraindication of arthroscopic surgery.

This study aims at: a) examining the visibility and morphology of the petrotympanic fissure on cone-beam compute tomography images, and b) investigating whether or not the petrotympanic fissure morphology is significantly affected by gender and age.

\section{MATERIAL AND METHODS Subjects}

From the archives of a certified imaging center in Amsterdam, The Netherlands, a series of randomly selected patients' CBCT examinations, performed in the period from 2011 to 2015 , was used for the purposes of this study. The CBCT examinations of interest concerned the following: a) evaluation of paranasal sinuses, b) evaluation of TMJ, c) facial trauma, d) orthodontics, and d) implant planning.

The employed randomization procedure comprised the following steps: 4 CBCT examinations were randomly selected for each calendar month, with one 
CBCT examination selected for each week. Because the selected CBCT examinations concerned the years 2011 - 2015 inclusive, the initial cohort was 5 years $x$ 12 months $\mathrm{x} 4$ weeks $=240 \mathrm{CBCT}$ examinations. One hundred and six CBCT examinations (age range: 13 to 83 years, mean age 47.4 years, standard deviation [SD] 15.9 years; interquartile range [IQR] 36.6 years) were eligible for inclusion in this study. The inclusion criteria were: a) the CBCT concerned the anatomical area of interest, that is, TMJ, the tympanic cavity, and the adjacent temporal bone; and b) complete absence of any movement or stripe artefacts. The resulting sample consisted of 56 males (mean age 47.82 [SD 14.7] years), and 50 females (mean age 46.94 [SD 17.4] years). Moreover, 212 TMJ/tympanic areas ( 2 for each of the 106 patients) were separately evaluated (Figure 1).

No clinical information was made available to the observers that might have biased their perception. In addition, the patient's identity was protected by codification. It is noted that for this type of study formal consent was not required.

\section{CBCT imaging}

All CBCT examinations were made by using the NewTom VGi CBCT unit (QR Srl., Verona, Italy), at $110 \mathrm{kVp}, 2-5 \mathrm{~mA}$ and 3.6-second exposure time. A reconstruction volume of $15 \times 15 \mathrm{~cm}$ and a voxel size of $0.3 \mathrm{~mm}$ were used. Reconstructions of the volumetric data set were created by employing the manufacturer's proprietary software NewTom Cone Beam 3-D Imaging, NNT version 5.10 (QR Srl., Verona, Italy).

\section{Evaluation of the CBCT images}

The area of interest (TMJ, tympanic cavity, and the adjacent temporal bone) was selected from the reconstruction volume. It was subsequently enhanced to provide a clear description of the subtle structures. The reconstructed images were examined by two observers: a) an oral and maxillofacial radiologist (K.S.) with 13 years' experience in CBCT image interpretation; and b) a general dental practitioner (R.S.) with one-year CBCT imaging training. Due to the complex anatomy of this region, the two observers assessed by consensus the morphology of PTF.

The protocol used is as follows: initially, the CBCT data-sets were viewed at axial planes, starting from the most cranial view. By scrolling down this continued until a clear view of the simultaneous presence of PTF, the adjacent mandibular fossa, and the cranial part of the tympanic cavity, was reached. Subsequently, cross-sectional images at an interval of $1 \mathrm{~mm}$ were created by adopting a modification of Pöschl's projection [23,24]. This modification entailed the creation of a level at an angle of $45^{\circ}$ approximately to the coronal and sagittal planes and aligned parallel to the PTF. These cross-sectional images exhibit the best display of the PTF, the mandibular fossa, and the tympanic cavity (Figure 2).

The PTF-morphology was categorized in three types: type 1 - widely open; type 2 - narrow middle; type 3 - very narrow/closed. These types were based on the morphological characteristics of the shape and the degree of closure, and they are similar to those described by Sato et al. [16]. Only the description of type 2 has been slightly modified (Figure 3 - 5).

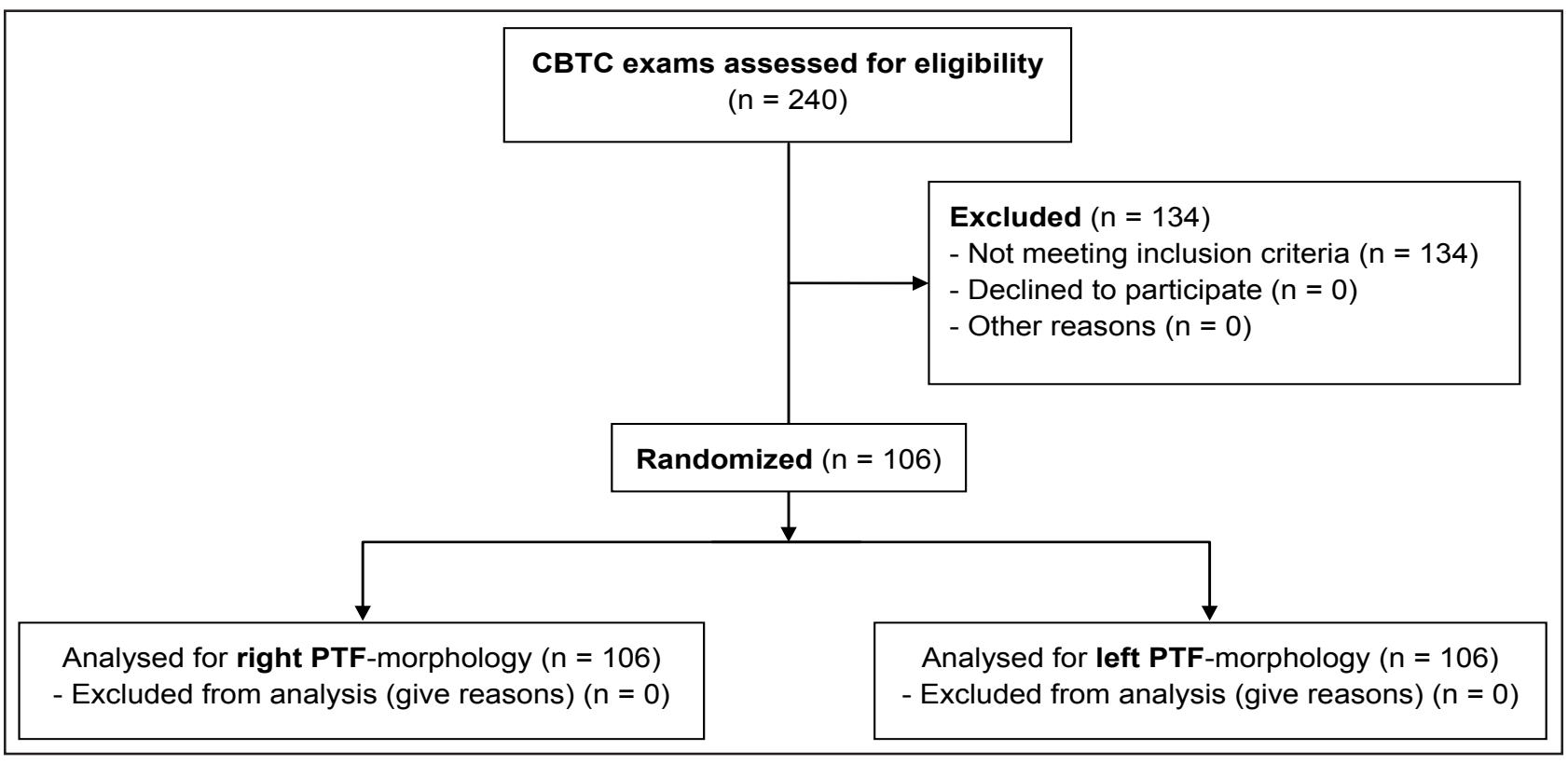

Figure 1. Enrolment process for the subjects [30]. 


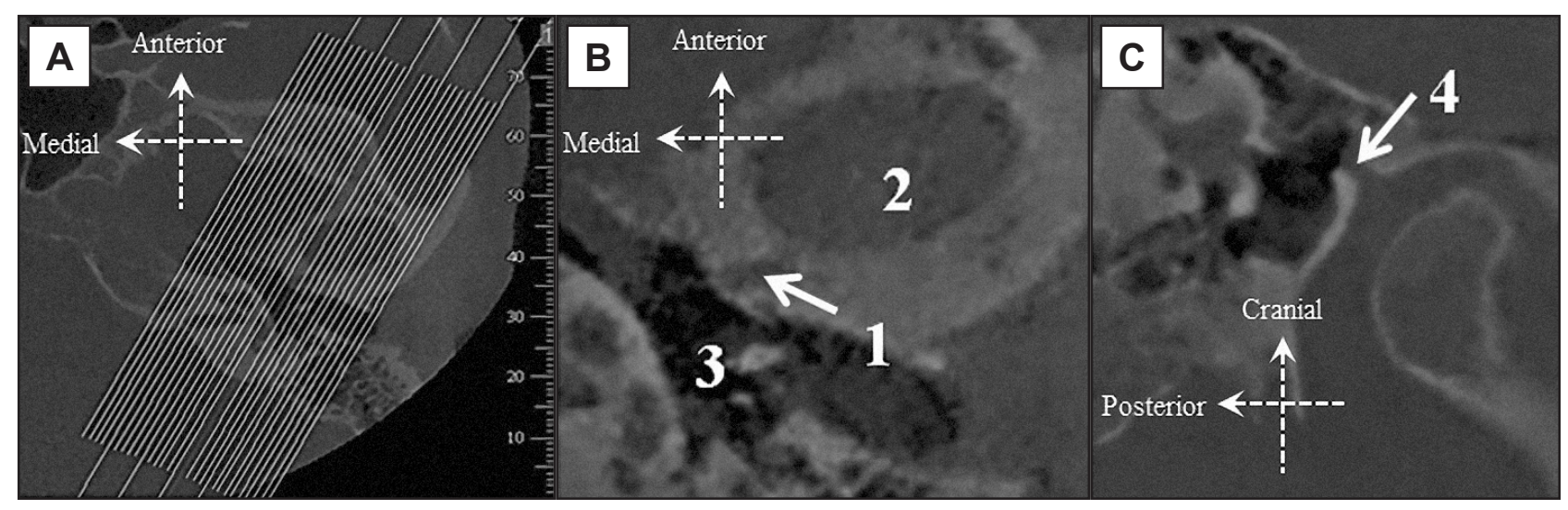

Figure 2. $\mathrm{A}=$ cross-sectional image setup according to the modification of the Pöschl projection. $\mathrm{B}=$ axial slice showing the petrotympanic fissure (1, arrow), mandibular fossa (2) and the tympanic cavity (3). $\mathrm{C}=$ cross-sectional display of the petrotympanic fissure (4, arrow).

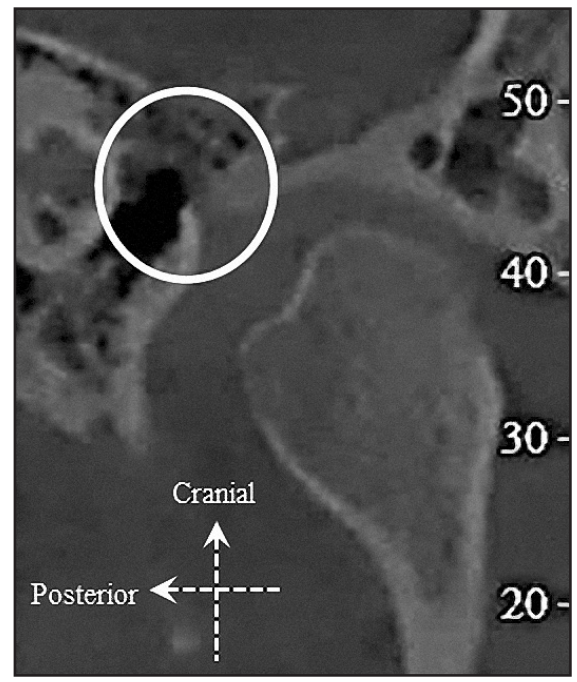

Figure 3. Cone-beam computed tomography image of the right TMJ area (sagittal plane), showing (circle) the petrotympanic fissure morphology type 1 (wide tunnel-shape).

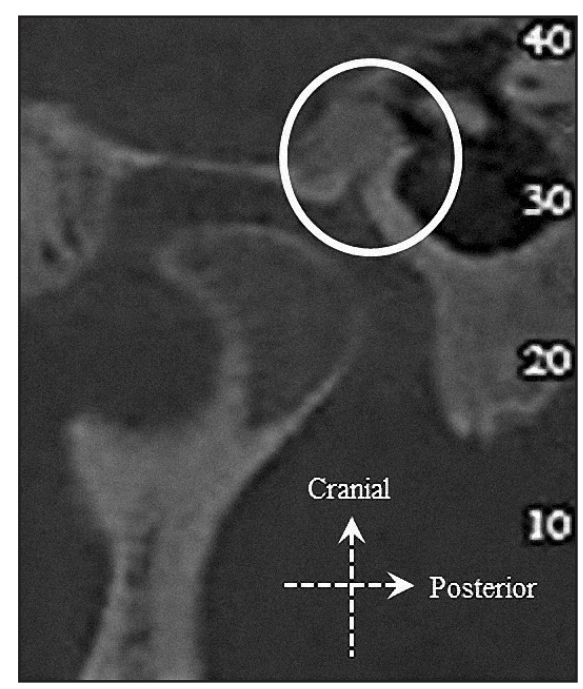

Figure 5. Cone-beam computed tomography image of the left TMJ area (sagittal plane), showing (circle) the petrotympanic fissure morphology type 3 (very narrow/closed tunnel-shaped.

Widely open at the entrance of the mandibular fossa, while the middle region resembles a flat-shaped tunnel structure and narrow (to almost closed) exit in the tympanic cavity).

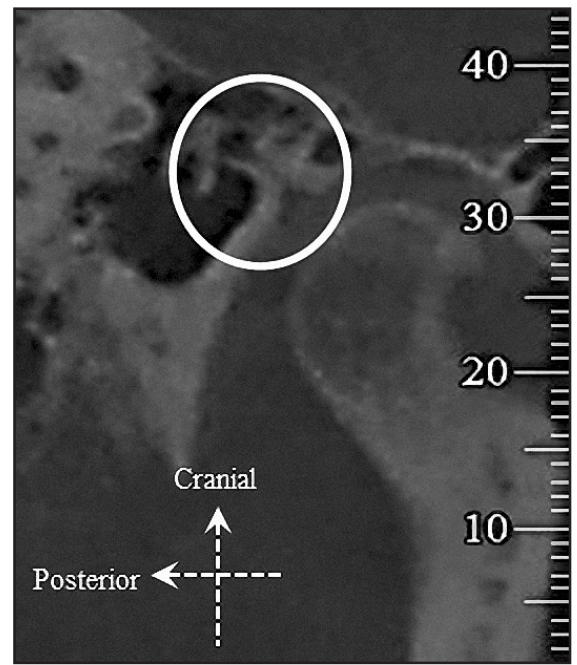

Figure 4. Cone-beam computed tomography image of the right TMJ area (sagittal plane), showing (circle) the petrotympanic fissure morphology type 2 (narrowed tunnel-shaped.

Widely open at the entrance of the mandibular fossa and open at the exit in the tympanic cavity, but narrow in the middle aspect).

\section{Statistical analysis}

Descriptive analysis was performed by summarizing categorical variables through counts and proportions $(\%)$, and continuous variables by their mean and standard deviation ( $\mathrm{M}[\mathrm{SD}])$. The association between left and right PTF-morphology was assessed by using the Cohen's weighted Kappa coefficient. Differences in PTF-morphology by gender and by age were summarised through two-way tables. The significance of these differences was assessed by using Chi-square tests or ordinal logistic regression models. Differences were considered significant when $\mathrm{P}<0.05$. All analyses have been performed by using Stata 13 (Stata Corp., TX USA).

\section{RESULTS}

The demographic characteristics of the sampled 
CBCTs of patients (gender and age) together with their PTF morphology are summarized in Table 1. Forty-seven out of the 106 CBCTs (44.3\%) exhibited the same type of PTF-morphology bilaterally. The resulting Kappa coefficient was 0.37 indicating a fair agreement, but a significant $(\mathrm{P}<0.001)$ association (Table 2).

Regarding the PTF morphology, no significant difference was found between males and females. Analysis with ordinal logistic regression showed a tendency for males to have narrower PTFs compared to females $(\mathrm{OR}=1.58$ for right and $\mathrm{OR}=1.5$ for left PTF), but gender was not found to play a significant role in PTF's morphology $(\mathrm{P}=0.264$ and $\mathrm{P}=0.211$ for right and left PTFs respectively) (Table 3 ).

To investigate further any potential correlation of the PTF's morphology with age, we created three sub-groups as a function of age: group A, $14-29$ years; group B, $30-49$ years; group C: $>50$ years. Results showed that the distribution of the right PTF's morphology was uniform across age groups $(\mathrm{P}=$ 0.04 ), whilst also the analysis through ordinal logistic regression did not reveal any systematic trends towards narrower or wider PTFs at older ages $(\mathrm{P}=$ 0.908). Similarly, there were no significant differences
$(\mathrm{P}=0.596)$ or trends $(\mathrm{P}=0.86)$ with increasing age in the distribution of the left PTF's morphology (Table 4).

Table 1. Demographic and clinical characteristics of the sample

\begin{tabular}{|c|c|}
\hline & N (\%) \\
\hline \multicolumn{2}{|l|}{ Gender } \\
\hline Female & $50(47.2)$ \\
\hline Male & $56(52.8)$ \\
\hline Total & $106(100)$ \\
\hline \multicolumn{2}{|l|}{ PTF morphology-right } \\
\hline Type 1 (widely open) & $47(44.3)$ \\
\hline Type 2 (narrow middle) & $28(26.4)$ \\
\hline Type 3 (very narrow/closed) & $31(29.2)$ \\
\hline \multicolumn{2}{|l|}{ PTF morphology-left } \\
\hline Type 1 (widely open) & $38(35.8)$ \\
\hline Type 2 (narrow middle) & $44(41.5)$ \\
\hline Type 3 (very narrow/closed) & $24(22.6)$ \\
\hline Total & $212(200)$ \\
\hline \multirow{2}{*}{ Age (years) } & Mean (SD) \\
\hline & $47.4(15.9)$ \\
\hline
\end{tabular}

$\mathrm{PTF}=$ petrotympanic fissure $\mathrm{N}=$ number; $\mathrm{SD}=$ standard deviation.

Table 2. Distribution of the PTF morphology-right, within the PTF morphology-left categories

\begin{tabular}{|c|c|c|c|c|c|}
\hline & \multicolumn{3}{|c|}{ PTF morphology-left } & \multirow{2}{*}{ Overall } & \multirow{3}{*}{ P-value } \\
\hline & Widely open & Narrow middle & Very narrow/closed & & \\
\hline & $\mathbf{N}(\%)$ & N (\%) & $\mathbf{N}(\%)$ & N (\%) & \\
\hline \multicolumn{6}{|l|}{ PTF morphology-right } \\
\hline Type 1 (widely open) & $26(68.4)$ & $15(34.1)$ & $6(25)$ & $47(44.3)$ & \multirow{4}{*}{0.001} \\
\hline Type 2 (narrow middle) & $9(23.7)$ & $11(25)$ & $8(33.3)$ & $28(26.4)$ & \\
\hline Type 3 (very narrow/closed) & $3(7.9)$ & $18(40.9)$ & $10(41.7)$ & $31(29.2)$ & \\
\hline Total & $38(100)$ & $44(100)$ & $24(100)$ & $106(100)$ & \\
\hline
\end{tabular}

$\mathrm{PTF}=$ petrotympanic fissure; $\mathrm{N}=$ number.

Table 3. Distribution of the PTF's morphology (left and right) by gender.

\begin{tabular}{|c|c|c|c|c|}
\hline & $\begin{array}{c}\text { Female }^{\mathrm{a}} \\
(\mathrm{n}=50 ; 47.2 \%)\end{array}$ & $\begin{array}{c}\text { Male }^{\mathrm{a}} \\
(\mathrm{n}=56 ; 52.8 \%)\end{array}$ & $\begin{array}{c}\text { Overall }^{\mathrm{a}} \\
(\mathrm{n}=106 ; 100 \%)\end{array}$ & P-value \\
\hline \multicolumn{5}{|l|}{ PTF morphology-right } \\
\hline Type 1 (widely open) & $25(50)$ & $22(39.3)$ & $47(44.3)$ & \multirow{3}{*}{$0.264^{\mathrm{b}}$} \\
\hline Type 2 (narrow middle) & $13(26)$ & $15(26.8)$ & $28(26.4)$ & \\
\hline Type 3 (very narrow/closed) & $12(24)$ & $19(33.9)$ & $31(29.2)$ & \\
\hline \multicolumn{5}{|l|}{ PTF morphology-left } \\
\hline Type 1 (widely open) & $20(40)$ & $18(32.1)$ & $38(35.8)$ & \multirow{3}{*}{$0.211^{\mathrm{b}}$} \\
\hline Type 2 (narrow middle) & $21(42)$ & $23(41.1)$ & $44(41.5)$ & \\
\hline Type 3 (very narrow/closed) & $9(18)$ & $15(26.8)$ & $24(22.6)$ & \\
\hline
\end{tabular}

${ }^{\mathrm{a}}$ All figures are $\mathrm{N}(\%)$ for PTF morphology.

${ }^{b}$ Not significant, ordinal logistic regression analysis test (significance level $\mathrm{P}<0.05$ ).

$\mathrm{PTF}=$ petrotympanic fissure; $\mathrm{n}=$ number . 
Table 4. Distribution of the PTF's morphology (left and right) by age group.

\begin{tabular}{|c|c|c|c|c|c|}
\hline & \multicolumn{3}{|c|}{ Age (years) } & \multirow{2}{*}{ Overall } & \multirow{3}{*}{ P-value } \\
\hline & $14-29$ & $30-49$ & $50+$ & & \\
\hline & $\mathbf{N}(\%)$ & $\mathbf{N}(\%)$ & $\mathbf{N}(\%)$ & $\mathbf{N}(\%)$ & \\
\hline \multicolumn{6}{|l|}{ PTF morphology - right } \\
\hline Type 1 (widely open) & $4(21.1)$ & $19(55.9)$ & $24(45.3)$ & $47(44.3)$ & \multirow{3}{*}{$0.04^{\mathrm{a}}$} \\
\hline Type 2 (narrow middle) & $8(42.1)$ & $10(29.4)$ & $10(18.9)$ & $28(26.4)$ & \\
\hline Type 3 (very narrow/closed) & $7(36.8)$ & $5(14.7)$ & $19(35.8)$ & $31(29.2)$ & \\
\hline \multicolumn{6}{|l|}{ PTF morphology - left } \\
\hline Type 1 (widely open) & $5(26.3)$ & $15(44.1)$ & $18(34)$ & $38(35.8)$ & \multirow{3}{*}{$0.596^{\mathrm{b}}$} \\
\hline Type 2 (narrow middle) & $9(47.4)$ & $14(41.2)$ & $21(39.6)$ & $44(41.5)$ & \\
\hline Type 3 (very narrow/closed) & $5(26.3)$ & $5(14.7)$ & $14(26.4)$ & $24(22.6)$ & \\
\hline Total & $19(100)$ & $34(100)$ & $53(100)$ & $106(100)$ & \\
\hline
\end{tabular}

${ }^{a}$ Significant, Chi-square test (significance level $\mathrm{P}<0.05$ ).

bNot significant, Chi-square test (significance level $\mathrm{P}<0.05$ ).

$\mathrm{PTF}=$ petrotympanic fissure; $\mathrm{N}=$ number.

\section{DISCUSSION}

This study lends significant support to the following: a) CBCT is sufficient to depict subtle structures as those of PTF; b) CBCT provides detailed information about the morphological characteristics of PTF; c) PTF-morphology is not related to age and gender; and d) the analysed CBCT images indicate that there is a tendency for males to have narrower PTFs than females.

Our results are in line with those of Çakur et al. $[\underline{18}, \underline{25}]$ who also found that there is no predisposition of age and gender to PTF-morphology. However, the incidence of the morphological types of PTF in our study differs from both the findings of Sato et al. [16] and also those of Çakur et al. [18,25] since both authors used the same classification pattern. The differences between this study and the studies of those two authors may, at least partly, be attributed to: a) the different cohorts studied, since Sato et al. [16] examined fourteen resected cadavers and Çakur et al. $[18,25] \mathrm{TMD} /$ tinnitus patients; and b) the different projections used, since they both used the Reid's base line as a reference level, and performed their measurements only at an anterior-posterior orientation on sagittal images. Interestingly, the incidence of the PTF-morphology's types differs significantly between the two studies of Çakur et al. $[\underline{18}, 25]$. In the present study, and as described by Sato et al. [16], a small modification of the types of PTF was applied. This modification was chosen to achieve a clear difference in terms of the radiographic display between the type 2 (narrow middle) and type 3 (very narrow/closed) PTF-morphology. Notably, regarding the type 2 (narrow middle) PTF-morphology our modification did not affect the final results, as these are comparable with those in the study of Chakur et al. [18]. The modification of Pöschl's projection $[23,24]$ adopted in the present study enables the clear observation of the PTF morphology across its entire length. This is due to the evaluation of the cross-sectional images - reconstructed from the volumetric data set - of the region of interest. These particular manipulations of the multi-planar CBCT imaging allow a clear and easy evaluation of this subtle structure, and not merely the sagittal images that was performed in the abovementioned studies.

One may criticise that the $\mathrm{CBCT}$ imaging contribution to the PTF evaluation might be questionable. The counter-argument to such criticism is that there are studies showing that CBCT provides better definition of fine osseous structures of temporal bone than that of currently available MSCT scanners [21]. Moreover, $\mathrm{CBCT}$ proved to be at least as accurate as routinely used MSCT in revealing the clinically and surgically important middle-ear structures [22]. Moreover, the study of Sato et al. [16] incorporates CBCT images in relation to data obtained from dissected cadavers mainly of the surrounding area of PTF. Thus, we believe that our choice of CBCT imaging is justified. Moreover, one further criticism of our study might be that we have not obtained concrete measurements on the types of PTFs, since our results have relied upon on visual evaluation only. However, the adopted modification of Pöschl's projection $[23,24]$ that we used provides a "volumetric" approach of the PTF morphology that is difficult to measure. It should also be noted that the magnification of the image may introduce inaccuracies that poses challenges 
to quantification. This could be the subject of future studies.

Another interesting finding of the present study is that there is no correlation between on the one hand age and gender, and on the other hand PTF-morphology. However, the incidence of the morphological types of PTF that were identified in our study could contribute to further clinical and/or pre-surgical evaluation of a patient as described below.

In view of the findings about the ligamentous connection that exists between the bilaminar zone of the articular disk of TMJ (posterior segment of the capsule of TMJ) and the tympanic cavity $[6,12,14-$ 16,26], the imaging techniques of the present study could contribute to the further evaluation of TMD related symptoms (e.g. anterior articular disk displacement, tinnitus, etc.) $[\underline{14}, \underline{16}, \underline{26}]$. This consideration is in agreement with Sato's et al. [16] suggestion that the movement of the malleus in the middle ear by traction of the DML depends on the degree of closure of the PTF during development. Also, Aristeguieta et al. [26] support this finding. At any rate, it must be borne in mind that the degree of closure of the PTF and its role in TMD and auditory symptoms development is still debated due to their multifactorial aetiology.

Additionally, several TMJ surgical procedures such as: inferior distraction of the condyle, surgery for internal derangements, condylar trauma, and vertical distraction of the mandibular ramus, may cause stretching of these ligamentous structures resulting in disturbing the middle ear ossicles equilibrium [12].

The incidence of $44.3 \%$ type 1 - widely open PTF at the right side and $35.8 \%$ type 1 - widely open PTF at the left side should be taken into consideration when planning an arthroscopic surgery. Our findings are in agreement with the surgical recommendations of McCain [27] about avoiding complications when performing arthroscopic surgery. Tsuyama et al. [] pointed out that the operator should have a thorough understanding of the regional anatomy involved to prevent complications arising from TMJ arthroscopic surgery. Our results contribute to this effort. Thus, by using $\mathrm{CBCT}$ data, the surgeon will have a clear view, first, of the fine bony structure laying underneath the navigation points used for arthroscopy, and, second, the agenesis of the foramen of Huschke, which although rare, it could nonetheless be a potential site of entrance into the middle ear from the posterior aspect of the TMJ.

At any rate, when sharp instruments are used for penetrating the joint, these should be directed anteriorly. Judicious use of mini-instruments could enhance safe arthroscopic surgery [13]. Both mechanical ruptures of the joint structures caused by the surgical and arthroscopic instruments and also fluid leaks due to high pressure irrigation could be the cause of otologic complications [료, 29] .

\section{CONCLUSIONS}

This study supports that particular manipulations of the multi-planar cone-beam computed tomography imaging enable a clear depiction of the morphological characteristics of the subtle structure of petrotympanic fissure. We have found that its morphology is neither gender nor age related. Due to the limitations of our study, the results should be taken with caution. At any rate, they could contribute to the preoperative evaluation of the petrotympanic fissure morphology, thereby contributing to the avoidance of complications when performing arthroscopic surgery.

\section{ACKNOWLEDGMENTS AND DISCLOSURE STATEMENTS}

The authors report no conflicts of interest related to this study. Also, this research did not receive any specific grant from funding agencies in the public, commercial, or not-for-profit sectors.

\section{REFERENCES}

1. Van Sickels JE, Nishioka GJ, Hegewald MD, Neal GD. Middle ear injury resulting from temporomandibular joint arthroscopy. J Oral Maxillofac Surg. 1987 Nov;45(11):962-5. [Medline: 3312539] [doi: 10.1016/0278-2391(87)90450-2]

2. Applebaum EL, Berg LF, Kumar A, Mafee MF. Otologic complications following temporomandibular joint arthroscopy. Ann Otol Rhinol Laryngol. 1988 Nov-Dec;97(6 Pt 1):675-9. [Medline: 3202572] [doi: 10.1177/000348948809700618]

3. Carroll TA, Smith K, Jakubowski J. Extradural haematoma following temporomandibular joint arthrocentesis and lavage. Br J Neurosurg. 2000 Apr;14(2):152-4. [Medline: 10889893] [doi: 10.1080/02688690050004633]

4. Patel S, Jerjes W, Upile T, Hopper C. TMJ arthroscopy: rare neurological complications associated with breach of the skull base. Br J Oral Maxillofac Surg. 2010 Jun;48(4):e18-20. [Medline: 20362372] [doi: 10.1016/j.bjoms.2009.12.012]

5. Holmlund A, Hellsing G. Arthroscopy of the temporomandibular joint. An autopsy study. Int J Oral Surg. 1985 Apr;14(2):169-75. [Medline: 3920161] [doi: 10.1016/S0300-9785(85)80089-2] 
6. Tsuyama M, Kondoh T, Seto K, Fukuda J. Complications of temporomandibular joint arthroscopy: a retrospective analysis of 301 lysis and lavage procedures performed using the triangulation technique. J Oral Maxillofac Surg. 2000 May;58(5):500-5; discussion 505-6. [Medline: 10800905] [doi: 10.1016/S0278-2391(00)90010-7]

7. González-García R, Gil-Díez Usandizaga JL, Rodríguez-Campo FJ. Arthroscopic anatomy and lysis and lavage of the temporomandibular joint. Atlas Oral Maxillofac Surg Clin North Am. 2011 Sep;19(2):131-44. [Medline: 21878248] [doi: 10.1016/i.cxom.2011.05.002]

8. Sugisaki M, Ikai A, Tanabe H. Dangerous angles and depths for middle ear and middle cranial fossa injury during arthroscopy of the temporomandibular joint. J Oral Maxillofac Surg. 1995 Jul;53(7):803-10. [Medline: 7595795] [doi: 10.1016/0278-2391(95)90337-2]

9. Pinto OF. A new structure related to the temporomandibular joint and middle ear. J Prosthet Dent. 1962 JanFeb;12(1):95-103. [doi: 10.1016/0022-3913(62)90014-8]

10. Cheynet F, Guyot L, Richard O, Layoun W, Gola R. Discomallear and malleomandibular ligaments: anatomical study and clinical applications. Surg Radiol Anat. 2003 May;25(2):152-7. [Medline: 12802513] [doi: 10.1007/s00276-003-0097-y]

11. Rodríguez-Vázquez JF, Murakami G, Verdugo-López S, Abe S, Fujimiya M. Closure of the middle ear with special reference to the development of the tegmen tympani of the temporal bone. J Anat. 2011 Jun;218(6):690-8. [Medline: 21477146] [doi: 10.1111/j.1469-7580.2011.01378.x]

12. Sencimen M, Varol A, Baykal B, Altug HA, Dogan N, Sahin S, Okcu KM, Yalcin B. Histological Characteristics of Ligaments between Middle Ear and Temporomandibular Joint. Eur J Dent. 2009 Oct;3(4):280-4. [Medline: 19826599] [doi: 10.1016/j.bjoms.2009.06.203]

13. Machon V, Levorova J, Foltan R, Hirjak D, Sidebottom A. Mini-instruments for minimally invasive arthroscopy of the temporomandibular joint: a technical note. Br J Oral Maxillofac Surg. 2015 Sep;53(7):662-3. [Medline: 26060135] [doi: 10.1016/j.bjoms.2015.04.015]

14. Mérida-Velasco JR, de la Cuadra-Blanco C, Pozo Kreilinger JJ, Mérida-Velasco JA. Histological study of the extratympanic portion of the discomallear ligament in adult humans: a functional hypothesis. J Anat. 2012 Jan;220(1):86-91. [Medline: 22050648] [doi: 10.1111/j.1469-7580.2011.01447.x]

15. Rowicki T, Zakrzewska J. A study of the discomalleolar ligament in the adult human. Folia Morphol (Warsz). 2006 May;65(2):121-5. [Medline: 16773599 ]

16. Sato I, Arai H, Asaumi R, Imura K, Kawai T, Yosue T. Classifications of tunnel-like structure of human petrotympanic fissure by cone beam CT. Surg Radiol Anat. 2008 Jun;30(4):323-6. [Medline: 18288438] [doi: 10.1007/s00276-008-0327-4]

17. Buergers R, Kleinjung T, Behr M, Vielsmeier V. Is there a link between tinnitus and temporomandibular disorders? J Prosthet Dent. 2014 Mar;111(3):222-7. [Medline: 24286640] [doi: 10.1016/j.prosdent.2013.10.001]

18. Çakur B, Yaşa Y. Correlation Between Tinnitus and Petrotympanic Fissure Status Among Patients With Temporomandibular Joint Dysfunction. J Oral Maxillofac Surg. 2016 Jan;74(1):47-52. [Medline: 26279489] [doi: 10.1016/i.joms.2015.07.020]

19. Schickinger B, Gstoettner W, Cerny C, Kornfehl J. Variant petrotympanic fissure as possible cause of an otologic complication during TMJ arthroscopy. A case report. Int J Oral Maxillofac Surg. 1998 Feb;27(1):17-9. [Medline: 9506293] [doi: 10.1016/S0901-5027(98)80089-1]

20. Anagnostopoulou S, Venieratos D, Antonopoulou M. Temporomandibular joint and correlated fissures: anatomical and clinical consideration. Cranio. 2008 Apr;26(2):88-95. [Medline: 18468268] [doi: 10.1179/crn.2008.013]

21. Gupta R, Bartling SH, Basu SK, Ross WR, Becker H, Pfoh A, Brady T, Curtin HD. Experimental flat-panel high-spatialresolution volume CT of the temporal bone. AJNR Am J Neuroradiol. 2004 Sep;25(8):1417-24. [Medline: 15466345]

22. Peltonen LI, Aarnisalo AA, Kortesniemi MK, Suomalainen A, Jero J, Robinson S. Limited cone-beam computed tomography imaging of the middle ear: a comparison with multislice helical computed tomography. Acta Radiol. 2007 Mar;48(2):207-12. [Medline: 17354143] [doi: 10.1080/02841850601080465]

23. Ozgen B, Cunnane ME, Caruso PA, Curtin HD. Comparison of 45 degrees oblique reformats with axial reformats in CT evaluation of the vestibular aqueduct. AJNR Am J Neuroradiol. 2008 Jan;29(1):30-4. [Medline: 17947373] [doi: 10.3174/ajnr.A0735]

24. Pöschl M. [Der tomographische Querschnitt durch das Felsenbein]. Fortschritte auf der Gebiete des Rontgenstrahlen 1943;68:174-79.

25. Çakur B, Sümbüllü MA, Durna D,Akgül HM. Prevalence of the types of the petrotympanic fissure in the temporomandibular joint dysfunction. Acta Radiol. 2011 Jun 1;52(5):562-5. [Medline: 21498294] [doi: 10.1258/ar.2011.100409]

26. Aristeguieta LMR, Acuña LEB, Ortiz GPS. A direct anatomical study of the morphology and functionality of discomalleolar and anterior malleolar ligaments. Int J Morphol. 2009;27(2):367-79. [doi: 10.4067/S0717-95022009000200011]

27. McCain JP, Sanders B, Koslin MG, Quinn JH, Peters PB, Indresano AT. Temporomandibular joint arthroscopy: a 6-year multicenter retrospective study of 4,831 joints. J Oral Maxillofac Surg. 1992 Sep;50(9):926-30. [Medline: 1506966] [doi: 10.1016/0278-2391(92)90047-4]

28. Jones JL, Horn KL. The effect of temporomandibular joint arthroscopy on ear function. J Oral Maxillofac Surg. 1989 Oct;47(10):1022-5. [Medline: 2795293] [doi: 10.1016/0278-2391(89)90172-9] 
29. Vaira LA, Soma D, Meloni SM, Dellàversana Orabona G, Piombino P, De Riu G. Vertiginous crisis following temporomandibular joint athrocentesis: a case report. Oral Maxillofac Surg. 2017 Mar;21(1):79-81. [Medline: 27924428] [doi: 10.1007/s10006-016-0603-0]

30. Schulz KF, Altman DG, Moher D; CONSORT Group. CONSORT 2010 statement: updated guidelines for reporting parallel group randomised trials. BMJ. 2010 Mar 23;340:c332. [Medline: 20332509] [PMC free article: 2844940] [doi: $10.1136 /$ bmj.c332]

\section{To cite this article:}

Damaskos S, Syriopoulos K, Sens RL, Politis C.

An Investigation of the Morphology of the Petrotympanic Fissure Using Cone-Beam Computed Tomography

J Oral Maxillofac Res 2018;9(1):e4

URL: http://www.ejomr.org/JOMR/archives/2018/1/e4/v9n1e4.pdf

doi: $\underline{10.5037 / \text { jomr.2018.9104 }}$

Copyright $\mathbb{C}$ Damaskos S, Syriopoulos K, Sens RL, Politis C. Published in the JOURNAL OF ORAL \& MAXILLOFACIAL RESEARCH (http://www.ejomr.org), 31 March 2018.

This is an open-access article, first published in the JOURNAL OF ORAL \& MAXILLOFACIAL RESEARCH, distributed under the terms of the Creative Commons Attribution-Noncommercial-No Derivative Works 3.0 Unported License, which permits unrestricted non-commercial use, distribution, and reproduction in any medium, provided the original work and is properly cited. The copyright, license information and link to the original publication on (http://www.ejomr.org) must be included. 